\title{
Opting for DBS: the role of patients' associations between scientific and humanistic knowledge
}

\section{Lucilla Bossi* \\ Parkinson Italia, Milan, Italy \\ *Correspondence: lucilla.bossi@fastwebnet.it}

The movement which, in the years between the late 1980s and the early 1990s of the past century, led to the establishment of the first patients' associations in Italy was initially based on the patients' need for adequate medical information. In those days, upon leaving the neurologist's office with a diagnosis of Parkinson's disease (PD), you were stuck with the feeling of having received a really bad piece of news while - at the same time - not having the tools necessary to fully understand such diagnosis and its impact on your life. The adjustment to the new situation, which still nowadays can take many years, then was made even harder by the difficulty of gathering the information needed to understand what this illness really was and what its causes, its progression and outcomes were.

However, today a doubt is creeping among the associations: that they are going from one extreme to the other. As a matter of fact, it is a common occurrence to discover that too much information has been made available to the patients. Among the most common signs of information overload is the patients' fear and overreaction to the prospected future course of the condition: therefore, they quite often end up assuming the "omnipotent" illusion that the more they know about the disease, the easier it will be to be in control of it.

Considering that nowadays the information to the patients is taken for granted, it may be worth finding out if there are other kinds of knowledge the associations could take care of that might prove to be useful in order to improve the patient's quality of life. From this perspective the associations could be of real service if they broadened their mission to include a humanistic knowledge attentive not only to the physical needs of the suffering human beings but also to the emotional, intellectual, and spiritual ones. Such knowledge could be of great help to the people with Parkinson's, supporting them in critical situations such as the agonizing decision making process leading to the resolution to undergo deep brain stimulation (DBS) or drop the idea. The simple possibility of brain surgery evokes ghastly pictures of devastating lobotomies which leave the patient in the grip of anxiety and distress. And desperately alone. For however close and loving his/her family may be, however caring his friends and competent his doctors, the head that 1 day will have to be screwed into the stereotactic helmet is the patient's one. As his/her is the brain that all life long will depend for "normal" functioning on a constant electric charge. Moreover, the association offers the candidate an invaluable opportunity for the operation: getting in touch with other patients who have already undergone DBS or plan to do so. Thanks to the association, the patient gets acquainted with a wide range of human situations and individual reactions, while finding some relief from anxiety and attaining a more serene outlook.

This function of the associations could be implemented if, to help their members, they could offer them, besides well trained wholehearted volunteers, the help of a philosophical counselor. This is a new professional figure - drawing his knowledge from the more than 2500-year-old western philosophy - which has existed for some time now in more than a few Italian hospitals. The difference between a philosophical counselor and a psychologist lies herein: while psychotherapy addresses the patients unease from the point of view of his personal story and situation, philosophical counseling helps the patients to adjust to circumstances which, even though striking individually, are universal (i.e., depending on the human condition as such).

In my capacity as President of Parkinson Italia - a Federation of 23 patients' associations - I think that the availability of a qualified philosophical counselor, both as a group leader and for individual sessions would make a remarkable shift in the associations' role: from simple information provider to a place of warm reception and inner growth.

In the context of a wider understanding of the meaning of suffering it is worth noticing that according to ancient wisdom, disease is not only a setback but an opportunity as well, fostering meaningful insights into our inner world and taking stock of our life. A long disease gives us time to inquire ourselves in depth about many issues that otherwise would have remained buried deeply in our unconscious. Then the disease becomes a descent in the innermost depths of the soul. And it is just when suffering grows unbearable and we feel that we can not hold on for one more single second that the quantum leap takes place and suddenly the consciousness opens to a new perception of the world. But unfortunately our culture is one of appearance and consumerism, while experiencing the sacred is considered an embarrassing occurrence. But human beings do need spiritual nourishment especially if they are about to pass through "the narrow doors" of experience. And among the many "tight spots" trough which the disease forces us, brain surgery is possibly one of the narrowest and of the most crowded with unanswered questions.

One would expect from a patient restored to a better life at least some contentment. On the contrary there are more than a few people with Parkinson's who, after a completely successful DBS which greatly improved their motor symptoms, instead of being happy develop a depression or other psychiatric conditions.

It is clear that the above problem is deeply hidden and neurologists, neurosurgeons, and psychiatrists look for a solution in a specific malfunctioning area of the brain. Even in a perspective that does not reduce the psyche to a simple epiphenomenon of brain activity, one must admit that the fact is unsettling and raises many questions. How is it possible that infirmity 
could be regretted by a person to whom DBS gave back the priceless good of autonomy? What unimaginable and possibly perverse secondary gains could hide in such a distressing disease? What secret reward could be worth the agony of advanced Parkinson's.

However it must be remembered that recently it has been supposed that some of the psychiatric problems following surgery could be related to the sudden reduction of the dopaminergic drugs that usually takes place in the follow up.
It remains that there is no certainty at all and that such questions are not easily answered.

It is a dangerous ground where both humanistic and scientific knowledge are tightly interwoven together with ethics; however the resulting pattern is still blurred and indistinct.

As for me, treated by DBS with extraordinary results, the feeling is that we are just at the very beginning but, at the same time, that we are bound to reach most encouraging goals. As I used to say at the time of my operation: "It may-not be a cure, but it certainly feels much like one.”

Received: 07 January 2011; accepted: 18 April 2011; published online: 02 May 2011.

Citation: Bossi L (2011) Opting for DBS: the role of patients' associations between scientific and humanistic knowledge. Front. Integr. Neurosci. 5:11. doi: 10.3389/fnint.2011.00011 Copyright $\odot 2011$ Bossi. This is an open-access article subject to a non-exclusive license between the authors and Frontiers Media SA, which permits use, distribution and reproduction in other forums, provided the original authors and source are credited and other Frontiers conditions are complied with. 